\title{
Características clinicopatológicas de nódulos pulmonares: Experiencia en Clínica Reina Sofía, Bogotá, Colombia
}

\author{
Clinicopathological characteristics of pulmonary nodules: Experience at \\ Clínica Reina Sofía, Bogotá, Colombia
}

\author{
Alexander Fernández-Arrieta' ${ }^{\mathbb{D}}$, Stella Isabel Martinez-Jaramillo² $\mathbb{D}$, \\ Angie Carolina Riscanevo-Bobadilla ${ }^{3} \mathbb{D}$, Laura Lucia Escobar-Avila ${ }^{4} \mathbb{D}$
}

1 Médico, especialista en Cirugía general, fellow Cirugía de Tórax. Universidad El Bosque, Bogotá, D.C., Colombia.

2 Médica, especialista en Cirugía general y en Cirugía de Tórax; directora, programa de Cirugía de Tórax, Universidad El Bosque; Clínica Reina Sofía, Bogotá, D.C., Colombia.

3 Médica, residente de Cirugía General, Fundación Universitaria Sanitas, Bogotá, D.C., Colombia.

4 Médica, especialista en Patología, Clínica Reina Sofía, Bogotá, D.C., Colombia.

\section{Resumen}

Introducción. El cáncer de pulmón es la primera causa de mortalidad por cáncer a nivel mundial, lo que hace que sea considerado un problema de salud pública. Existen diferentes hallazgos imagenológicos que hacen sospechar la presencia de cáncer de pulmón, uno de los cuales son los nódulos pulmonares; sin embargo, estos también pueden verse en entidades benignas.

Métodos. Se incluyeron 66 pacientes con biopsia de nódulo pulmonar en la Clínica Reina Sofía, en la ciudad de Bogotá, D.C., Colombia, entre el $1^{\circ}$ de marzo del 2017 y el 28 de febrero del 2020. Se analizaron las características demográficas de los pacientes, las características morfológicas e histopatológicas de los nódulos pulmonares y la correlación entre sus características imagenológicas e histopatológicas.

Resultados. El 69,2 \% de los nódulos estudiados tenían etiología maligna, de estos el 55,5\% era de origen metástasico y el 44,5 \% eran neoplasias primarias de pulmón, con patrón sólido en el 70,6 \% de los casos. El patrón histológico más frecuente fue adenocarcinoma. Respecto a las características radiológicas, en su mayoría los nódulos malignos medían de 1 a 2 cm, de morfología lisa y distribución múltiple, localizados en lóbulos superiores.

Conclusiones. La caracterización de los nódulos pulmonares brinda información relevante que orienta sobre los diagnósticos más frecuentes en nuestro medio, cuando se estudian nódulos sospechosos encontrados incidentalmente o en el seguimiento de otro tumor. Como el nódulo es la manifestación del cáncer temprano del pulmón, establecer programas de tamización que permitan el diagnóstico oportuno, es hoy día una imperiosa necesidad, para reducir la mortalidad.

Palabras clave: neoplasias pulmonares; nódulo pulmonar solitario; biopsia con aguja; patología; diagnóstico; diagnóstico por imagen.

Fecha de recibido: 01/06/2021 - Fecha de aceptación: 30/09/2021 - Publicación en línea: 01/10/2021

Correspondencia: Alexander Fernández-Arrieta, Carrera 21 \# 29 B - 25, Edificio Bocas del Toro, Apto 702, Cartagena. Colombia.

Teléfono: 300573 6318. Correo electrónico: affernandez@unbosque.edu.co, affa1601@hotmail.com

Citar como: Fernández-Arrieta A, Martinez-Jaramillo SI, Riscanevo-Bobadilla AC, Escobar-Avila LL. Características clinicopatológicas de nódulos pulmonares: Experiencia en Clínica Reina Sofía, Bogotá, Colombia. Rev Colomb Cir. 2022;37:49-59.

https://doi.org/10.30944/20117582.903

Este es un artículo de acceso abierto bajo una Licencia Creative Commons - BY-NC-ND https://creativecommons.org/licenses/by-nc-nd/4.0/deed.es 


\begin{abstract}
Introduction. Lung cancer is the leading cause of cancer mortality worldwide, which makes it a public health problem. There are different imaging findings that suggest the presence of lung cancer, one of which is pulmonary nodules; however, these can also be seen in benign entities.

Methods. A total of 66 patients with pulmonary nodule biopsy at Clínica Reina Sofía, in the city of Bogotá D.C. were included between March 1, 2017 and February 28, 2020. The demographic characteristics of the patients, the morphologic and histopathologic characteristics of the pulmonary nodules and their correlation with their pathological diagnosis were analyzed.

Results. $69.2 \%$ of the nodules studied had malignant etiology, of these $55.5 \%$ were of metastatic origin, and $44.5 \%$ were primary lung neoplasms, with a solid pattern in $70.6 \%$ of the cases. The most frequent histological pattern was adenocarcinoma. Regarding the radiological characteristics, most of the malignant nodules measure 1 to $2 \mathrm{~cm}$, of smooth morphology and had multiple distribution, located in the upper lobes.

Conclusions. The characterization of pulmonary nodules provides relevant information that guides the most frequent diagnoses in our setting, when suspicious nodules found incidentally or in the follow-up of another tumor are studied. As the nodule is the manifestation of early lung cancer, establishing screening programs that allow timely diagnosis is an urgent need to reduce mortality.
\end{abstract}

Keywords: lung neoplasms; solitary pulmonary nodule; needle biopsy; pathology; diagnosis; diagnostic imaging.

\section{Introducción}

El cáncer de pulmón en la actualidad representa la principal causa de mortalidad por cáncer a nivel mundial y según el informe de Globocan 2020, la incidencia anual es de aproximadamente 2,2 millones de personas, lo que hace que sea considerado un problema de salud pública ${ }^{1,2}$. En Colombia se estima una prevalencia aproximada de 27,2 por 100.000 habitantes, con una tasa de incidencia calculada en hombres de 36,5 y en mujeres de 19,9 por 100.000 habitantes, correspondiendo a la tercera causa de muerte por cáncer en hombres y la quinta en mujeres ${ }^{2,3}$.

El cigarrillo ha sido identificado en el $90 \%$ de los casos como el principal factor de riesgo para el desarrollo de neoplasias de origen pulmonar ${ }^{4-7}$. Se ha estimado que el riesgo de desarrollar cáncer de pulmón en una persona fumadora es 20 veces mayor que en una persona no fumadora. La incidencia aumenta con la edad, exposición a otros agentes carcinogénicos como el radón, el asbesto, la exposición a radioterapia, la fibrosis pulmonar, factores genéticos, antecedentes de enfermedad pulmonar obstructiva crónica, hábito alcohólico e infección por VIH, entre otras ${ }^{8-13}$.
En la práctica clínica, los estadios tempranos pueden pasar desapercibidos debido a la ausencia de síntomas o signos, por lo que se hace necesaria la búsqueda activa con programas de tamización en pacientes con alto riesgo para cáncer de pulmón y la implementación simultánea de estrategias de prevención en los que se intervengan los factores de riesgo modificables, como el cese del tabaquismo.

Existen diferentes hallazgos radiológicos que hacen sospechar la presencia de cáncer de pulmón, y uno de estos son los nódulos pulmonares. Estos han sido definidos como opacidades con un diámetro de 3 centímetros o menos, rodeadas de parénquima pulmonar, pueden ser únicos o múltiples, sólidos o subsólidos, y pueden localizarse en uno o ambos pulmones. Las lesiones mayores de 3 centímetros, se denominan masas y poseen mayor probabilidad de ser malignas. Las características del nódulo y los factores de riesgo del paciente nos orientan a sospechar que un nódulo sea o no maligno.

Su evaluación radiológica mediante tomografía computarizada (TC) ha permitido una mejor caracterización de los nódulos ${ }^{13-15}$. Sin embargo, 
en la actualidad no existe un consenso acerca de su manejo y seguimiento, lo que dificulta el diagnóstico preciso y favorece el retraso en su diagnóstico ${ }^{4}$. Esto supone presentaciones en estadios avanzados, lo cual impacta no solo en el tratamiento sino en el pronóstico, con un aumento consecuente en la carga económica de la enfermedad ${ }^{16-20}$.

El propósito de este estudio fue describir los resultados obtenidos en nuestra institución al correlacionar las características clínicas y radiológicas de los nódulos pulmonares con los resultados histopatológicos, en una cohorte de pacientes con hallazgos imagenológicos de nódulos pulmonares sospechosos, sometidos a biopsia mediante abordaje multidisciplinar.

\section{Métodos}

Para el presente estudio se incluyeron todos los pacientes mayores de 18 años, con nódulos pulmonares que, en los registros de patología de la Clínica Reina Sofia, en la ciudad de Bogotá, D.C., Colombia, tuvieron biopsia de un nódulo pulmonar por cualquier método, entre el $1^{\circ}$ de marzo del 2017 y el 28 de febrero del 2020. Se excluyeron los pacientes con registros incompletos en la historia clínica.

Los datos fueron recolectados de manera retrospectiva. Se analizaron las características clínico-radiológicas, histológicas y anatomopatológicas de las lesiones y los métodos diagnósticos empleados, ya fueran biopsia transbronquial, percutánea o resección quirúrgica (cuñas o resecciones anatómicas).

Se hizo análisis para describir las características de la muestra, las variables cuantitativas se analizaron mediante medidas de tendencia central y dispersión; los datos categóricos mediante frecuencias y proporciones. Los supuestos de distribución normal en las variables de interés fueron evaluados con la prueba de Shapiro-Wilk y mediante análisis gráficos (histogramas de frecuencias y gráficos Q-Q).

Los pacientes fueron clasificados en dos grupos, con patología maligna o patología benigna, para el correspondiente análisis de las características radiológicas. Para calcular la proporción de las biopsias no quirúrgicas exitosas (aquellas realizadas a través de broncoscopia o biopsias percutáneas), se determinó la proporción de biopsias no quirúrgicas satisfactorias entre el total de biopsias no quirúrgicas realizadas. La mortalidad y las complicaciones se expresaron como el número total de pacientes que presentaron el evento (mortalidad o complicación) dentro de los 30 días posteriores a la cirugía. Los pacientes fueron evaluados en la consulta de control que se realiza aproximadamente entre los 7 y los 15 días después de realizado el procedimiento.

Se caracterizaron los nódulos pulmonares teniendo en cuenta su localización, tamaño, bordes y la presencia de calcificaciones o grasa, estabilidad o crecimiento; se utilizó la clasificación que los divide en sólidos y subsólidos ${ }^{9}$, estos últimos subdivididos en nódulos de vidrio esmerilado y los parcialmente sólidos, previamente denominados heterogéneos o mixtos.

\section{Resultados}

\section{Características demográficas de los pacientes}

Se seleccionó un total de setenta pacientes que cumplían con los criterios de inclusión y de ellos, se excluyeron 4 por no tener una historia clínica completa que permitiera su análisis. En total fueron elegibles para ser incluidos en el análisis sesenta y seis pacientes, en quienes se identificó la presencia de al menos un nódulo pulmonar mediante estudios imagenológicos y posteriormente fueron llevados a confirmación histopatológica.

De los 66 pacientes, 38 eran de sexo femenino (57,5 \%), la edad promedio fue de 68,1 años con desviación estándar de 13,6 (Rango: 24-89) (Tabla 1). Un total de 35 pacientes $(53,0 \%)$ tenían diagnóstico conocido de patología oncológica y 41 pacientes $(62,1 \%)$ antecedente familiar de patología oncológica al momento de la identificación del nódulo pulmonar; otros antecedentes personales patológicos fueron hipertensión arterial en 32 pacientes (48,4\%), diabetes mellitus en 8 pacientes $(12,1 \%)$ y enfermedad pulmonar en 9 pacientes $(13,6 \%)$. Otro antecedente relevante encontrado en los pacientes fue el hábito del tabaquismo en 23 pacientes $(34,8 \%)$. 
Tabla 1. Características generales y antecedentes relevantes de la muestra estudiada.

\begin{tabular}{lcc}
\hline & $\mathbf{N}$ & $\%$ \\
\hline Edad años (Rango) & $69(24-89)$ & \\
Sexo & & \\
$\quad$ Femenino & 38 & 57,5 \\
$\quad$ Masculino & 28 & 42,5 \\
Antecedente familiar oncológico & 41 & 62,1 \\
Antecedentes personales patológicos & & \\
$\quad$ Oncológico & 35 & 53,0 \\
$\quad$ Hipertensión arterial & 32 & 48,4 \\
$\quad$ Diabetes mellitus & 8 & 12,1 \\
Enfermedad pulmonar obstructiva & 5 & 7,6 \\
$\quad$ crónica (EPOC) & 4 & 6,0 \\
Otras enfermedades pulmonares & 4 & 6,0 \\
$\quad$ Enfermedad cardiovascular & 2 & 3,0 \\
$\quad$ Enfermedad del colágeno & 2 & 3,0 \\
$\quad$ Enfermedad renal crónica & & \\
Otros antecedentes relevantes & 23 & 34,8 \\
$\quad$ Fumador & & \\
\hline
\end{tabular}

Tabla 2. Diagnóstico histológico de los nódulos pulmonares

\begin{tabular}{|c|c|c|}
\hline Comportamiento histológico & $\mathbf{N}$ & $\%$ \\
\hline Benigno & 20 & 30,7 \\
\hline Inflamatorio & 9 & 45 \\
\hline Infeccioso & 6 & 30 \\
\hline Tumor benigno & 5 & 25 \\
\hline Sin evidencia de malignidad & 7 & \\
\hline Nueva biopsia & 6 & \\
\hline Observación & 1 & \\
\hline Maligno & 45 & 69,2 \\
\hline Metástasis & 25 & 55,5 \\
\hline Gastrointestinal & 9 & 20 \\
\hline Mama & 5 & 11,1 \\
\hline Sarcoma & 3 & 6,6 \\
\hline Genito-urinario & 2 & 4,4 \\
\hline Linfoma & 2 & 4,4 \\
\hline Próstata & 1 & 2,2 \\
\hline Fusocelular & 1 & 2,2 \\
\hline Ginecológico & 1 & 2,2 \\
\hline Parótida & 1 & 2,2 \\
\hline Primario & 20 & 44,5 \\
\hline Adenocarcinoma & 17 & 85,0 \\
\hline Acinar & 10 & 50,0 \\
\hline Sólido & 2 & 10,0 \\
\hline Lepídico & 2 & 10,0 \\
\hline In situ & 2 & 10,0 \\
\hline Mínimamente invasivo & 1 & 5,0 \\
\hline Neuroendocrinos & 2 & 10,0 \\
\hline Escamocelular & 1 & 5,0 \\
\hline
\end{tabular}

Dentro de las características clínicas se encontró la presencia de síntomas en la mitad de los pacientes, siendo la tos (36\%) el síntoma predominante, seguido de dolor torácico (14\%), disnea (11\%) y pérdida de peso $(6,3 \%)$.

El análisis histopatológico mostró nódulo maligno en 69,2 \% y benigno en 30,7 \%. De los 45 nódulos malignos, 55,5 \% eran de origen metastásico y 44,5\% tumores primarios del pulmón (Tabla 2). El origen de las metástasis en orden de frecuencia fue gastrointestinal $20 \%$ (con predominio de colon y recto), mama 11,1 \% y sarcomas en 6,6 \%. En los 20 pacientes donde el nódulo maligno correspondió a un tumor primario de pulmón, el diagnóstico más frecuente fue adenocarcinoma en el $85 \%$ (17/20).

Los nódulos fueron múltiples en el 57,5 \% y las localizaciones más frecuentes fueron los lóbulos superiores, con 12,1\% cada uno. Según el tipo de nódulo, fue sólido en el 77,2 \%, parcialmente sólido en el 12,2 \% y en vidrio esmerilado en el 10,6 \%. Otras características radiológicas del nódulo se describen en la tabla 3.

Un análisis adicional que comparó el tamaño del nódulo con el porcentaje de éxito de las biopsias mostró para el procedimiento percutáneo un éxito del $40 \%$ en los nódulos de 1 a $2 \mathrm{~cm}$ y del $75 \%$ en los mayores de $2 \mathrm{~cm}$; por su parte, en las biopsias transbronquiales, el éxito fue del 66,7 \% $(2 / 3)$ en los pacientes con los nódulos mayores de $2 \mathrm{~cm}$, correspondientes a un paciente con sarcoma y otro con adenocarcinoma metastásico gastrointestinal (Tabla 4).

$\mathrm{Al}$ analizar los 20 nódulos con histología benigna se encontró $45 \%$ de lesiones inflamatorias, $30 \%$ infecciosas, $25 \%$ tumores benignos y 7 pacientes sin evidencia de malignidad, 6 de los cuales fueron llevados posteriormente a biopsia quirúrgica para diagnóstico histológico definitivo, teniendo en cuenta que previo a esta intervención quirúrgica tenían características imagenológicas sospechosas de malignidad, y los resultados obtenidos fueron una adenoleiomiomatosis y dos tumores fibrosos de la pleura.

El paciente de la biopsia no conclusiva, que no aceptó una segunda intervención para confirmación histológica, ha tenido aproximadamente dos años de seguimiento radiológico y no ha presenta- 
Tabla 3. Características clínicas de los nódulos pulmonares

\begin{tabular}{|c|c|c|}
\hline & $\mathbf{N}$ & $\%$ \\
\hline Paciente presenta síntomas & 34 & 51,5 \\
\hline \multicolumn{3}{|l|}{ Evolución de los pacientes } \\
\hline Estudio inicial de nódulos & 51 & 77,2 \\
\hline Aumento de tamaño del nódulo & 11 & 16,6 \\
\hline Aparición de nuevos nódulos & 3 & 4,5 \\
\hline Sin datos & 1 & 1,5 \\
\hline \multicolumn{3}{|l|}{ Número de nódulos } \\
\hline Múltiples & 38 & 57,5 \\
\hline Únicos & 28 & 42,5 \\
\hline \multicolumn{3}{|l|}{ Localización de nódulos } \\
\hline Múltiples lóbulos & 36 & 54,8 \\
\hline Lóbulo superior derecho & 8 & 12,1 \\
\hline Lóbulo superior izquierdo & 8 & 12,1 \\
\hline Lóbulo medio & 5 & 7,5 \\
\hline Lóbulo inferior izquierdo & 5 & 7,5 \\
\hline Lóbulo inferior derecho & 4 & 6,0 \\
\hline \multicolumn{3}{|l|}{ Tipo de nódulo } \\
\hline Sólido & 51 & 77,2 \\
\hline Parcialmente sólido & 8 & 12,2 \\
\hline Vidrio esmerilado & 7 & 10,6 \\
\hline \multicolumn{3}{|l|}{ Características del nódulo } \\
\hline Liso & 22 & 33,3 \\
\hline Espiculado & 19 & 28,7 \\
\hline Lobulado & 19 & 28,7 \\
\hline Cavitado & 5 & 7,6 \\
\hline Calcificado & 1 & 1,5 \\
\hline \multicolumn{3}{|l|}{ Tamaño del nódulo } \\
\hline Menor de $1 \mathrm{~cm}$ & 12 & 18,1 \\
\hline De 1 a $2 \mathrm{~cm}$ & 38 & 57,5 \\
\hline Mayor de $2 \mathrm{~cm}$ & 16 & 24,2 \\
\hline
\end{tabular}

do cambios en la morfología y tamaño del nódulo pulmonar (Figura 1).

Al comparar el tipo y las características del nódulo según el comportamiento histológico (maligno o benigno), se encontró que los nódulos sólidos $(70,6 \%)$ y en vidrio esmerilado $(71,4 \%)$ son más frecuentemente malignos, mientras los parcialmente sólidos fueron malignos en el $50 \%$ de los casos.

Según las características morfológicas del nódulo (Tabla 5), predominaron los malignos, con mayor porcentaje en los lobulados en el $84,2 \%$ (16 pacientes) y los cavitados en el $80 \%$ (4 pacientes), mientras que el contorno liso fue la característica predominante entre los nódulos benignos, con 9 pacientes de 21 (40,9\%).

También se comparó el tipo del nódulo según la histopatología de tumor primario pulmonar, encontrando que los adenocarcinomas son principalmente sólidos (58,8\%), y en menor proporción en vidrio esmerilado $(23,5 \%)$ o parcialmente sólido $(17,7 \%)$. Todos los tumores neuroendocrinos y el escamocelular mostraron patrón sólido (Tabla 6).

Dentro de los aspectos relacionados con el diagnóstico se encontró que para la primera vía de abordaje el procedimiento realizado con mayor frecuencia fue la cirugía $(78,9 \%)$, seguido de la biopsia percutánea $(15,1 \%)$ y la biopsia transbronquial (6 \%) (Tabla 7). El éxito diagnóstico de las biopsias no quirúrgicas fue del $50 \%$; en el otro $50 \%$, correspondientes a 7 de 14 pacientes en los que no se logró el diagnóstico, en 6 se realizó como segundo procedimiento una biopsia quirúrgica, para obtener confirmación histológica, y el paciente restante se dejó en observación.

Tabla 4. Comparación del tamaño del nódulo con la realización y éxito de biopsia no quirúrgica

\begin{tabular}{lcccc}
\hline & \multicolumn{2}{c}{$\begin{array}{c}\text { Biopsia percutánea } \\
\mathbf{N = 1 0}\end{array}$} & \multicolumn{2}{c}{$\begin{array}{c}\text { Biopsia transbronquial } \\
\mathbf{N}=\mathbf{4}\end{array}$} \\
\cline { 2 - 5 } & \multicolumn{1}{c}{ Todas } & Éxito & Todas & Éxito \\
\hline Tamaño del nódulo & \multicolumn{3}{c}{0} \\
Menor de $1 \mathrm{~cm}$ & $1(9,0 \%)$ & $0(0,0 \%)$ & $0(0,0 \%)$ & $0(0,0 \%)$ \\
De 1 a $2 \mathrm{~cm}$ & $5(54,5 \%)$ & $2(40 \%)$ & $1(25,0 \%)$ & $0(0,0 \%)$ \\
Mayor de $2 \mathrm{~cm}$ & $4(36,4 \%)$ & $3(74,6 \%)$ & $3(75,0 \%)$ & $2(66,7 \%)$ \\
\hline
\end{tabular}




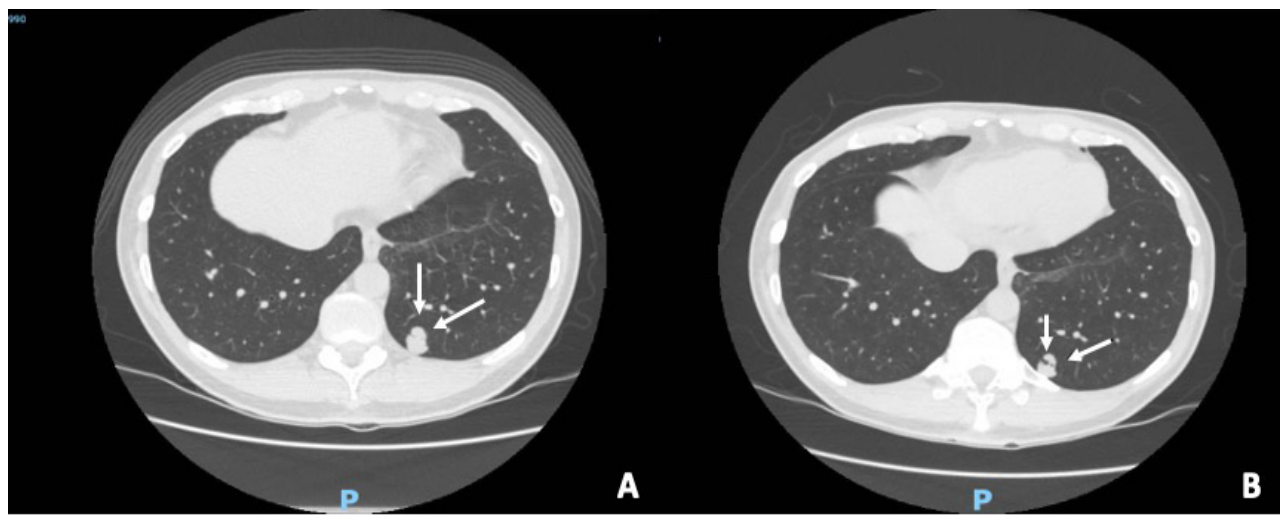

Figura. 1. Paciente en seguimiento de nódulo pulmonar. A. Tomografía axial computarizada del año 2018, donde se identificó un nódulo (flechas), al cual se realizó biopsia percutánea, con reporte de patología negativo para malignidad. B. Hallazgos imagenológicos del mismo nódulo pulmonar a los 2 años de seguimiento, sin cambios respecto a estudio previo.

Tabla 5. Características anatómicas del nódulo según comportamiento benigno o maligno

\begin{tabular}{lcc}
\hline & $\begin{array}{c}\text { Maligno } \\
\mathbf{N}=\mathbf{4 5}\end{array}$ & $\begin{array}{c}\text { Benigno } \\
\mathbf{N = 2 1 ^ { * }}\end{array}$ \\
\hline Características del nódulo & & \\
Liso & $13(59,1 \%)$ & $9(40,9 \%)$ \\
Espiculado & $12(63,5 \%)$ & $7(35,5 \%)$ \\
Lobulado & $16(84,2 \%)$ & $3(15,8 \%)$ \\
Cavitado & $4(80 \%)$ & $1(20 \%)$ \\
Calcificado & $0(0,0 \%)$ & $1(100 \%)$ \\
\hline
\end{tabular}

*20 confirmados por histología y uno que se asume por el seguimiento.

Tabla 6. Comportamiento del tipo de nódulo según histopatología de tumores primarios pulmonares.

\begin{tabular}{|c|c|c|c|}
\hline & $\begin{array}{c}\text { Adenocarcinoma } \\
n=17\end{array}$ & $\begin{array}{c}\text { Neuroendocrino } \\
n=2\end{array}$ & $\begin{array}{c}\text { Escamocelular } \\
n=1\end{array}$ \\
\hline \multicolumn{4}{|l|}{ Tipo de nódulo } \\
\hline Sólido & $10(58,8 \%)$ & $2(100 \%)$ & $1(100 \%)$ \\
\hline Parcialmente sólido & $3(17,7 \%)$ & $0(0,0 \%)$ & $0(0,0 \%)$ \\
\hline Vidrio esmerilado & $4(23,5 \%)$ & $0(0,0 \%)$ & $0(0,0 \%)$ \\
\hline
\end{tabular}

El tiempo promedio de estancia hospitalaria fue de 2,9 días (Rango 1-13). No hubo complicaciones intraoperatorias, pero en el $6 \%$ de los pacientes hubo complicaciones menores posteriores a la intervención quirúrgica, que no requirieron reintervención adicional mayor, consistente en
Tabla 7. Aspectos relacionados con el diagnóstico y evolución intrahospitalaria de los pacientes.

\begin{tabular}{lcc}
\hline & N & $\%$ \\
\hline Procedimiento realizado $1^{\text {a }}$ vía & 52 & 78.7 \\
Cirugía & 10 & 15.1 \\
Biopsia percutánea & 4 & 6 \\
Biopsia transbronquial & 59 & 89.4 \\
Diagnóstico por $1^{\text {a }}$ vía & 4 & 6.0 \\
Requiere UCl en el posoperatorio & 6 & 9.0 \\
Complicaciones & 4 & 6.0 \\
Menores (Fistula alveolopleural) & 2 & 3.0 \\
Mayores (Shock posoperatorio) & 60 & 91,1 \\
Ninguna & $2(1-13)$ \\
Días de estancia hospitalaria Me (Rango) & 2 & 3.0 \\
Mortalidad & 21 & 31.9 \\
Comportamiento Histológico & & \\
Maligno & 45 & 68.1 \\
Benigno & 21 \\
\hline
\end{tabular}

fístula alveolo-pleural que ocasionó enfisema subcutáneo, con escape persistente de aire en tres pacientes (uno de estos en combinación con neumotórax con requerimiento prolongado del tubo de tórax) y otro paciente con neumotórax menor del $30 \%$, que no afectaba la mecánica ventilatoria. 
Se requirió monitorización continua en unidad de cuidados intensivos en 4 (6\%) pacientes, dos de ellos con complicaciones mayores $(2,9 \%)$ que derivaron en mortalidad y los otros dos por la necesidad de vigilancia en el posoperatorio, uno de estos con acidosis respiratoria e hiperlactatemia leve después del procedimiento y otra paciente mujer de 82 años con múltiples comorbilidades que requirió una lobectomía media, ambos pacientes con posterior evolución satisfactoria.

En relación con la mortalidad, se presentó en dos de los pacientes, la primera una mujer de 86 años con carcinomatosis y derrame pleural maligno que se llevó al procedimiento diagnóstico en muy mal estado general, presentó en el postoperatorio inmediato actividad eléctrica sin pulso y falleció pocos días después, y el segundo paciente, un hombre de 76 años en quien la biopsia mediante una cuña de pulmón, demostró metástasis de neoplasia colorrectal conocida, requirió UCI por inestabilidad hemodinámica en el postoperatorio y se intervino nuevamente a las 12 horas descartando un sangrado como causa de su inestabilidad, quien falleció al décimo día postoperatorio a pesar de no tener complicaciones quirúrgicas.

\section{Discusión}

El hallazgo de nódulos pulmonares en estudios imagenológicos se convierte para el médico en un verdadero reto diagnóstico y genera ansiedad en el paciente, teniendo en cuenta que su etiología podría ser maligna. La aparición de cáncer de pulmón antes de los 30 años es muy rara y se incrementa con el aumento de la edad ${ }^{21-24}$. Los nódulos pulmonares en pacientes con edad mayor de 70 años han mostrado una mayor probabilidad de malignidad ${ }^{25,26}$, incluido el cáncer de pulmón. En nuestro estudio se identificó una edad promedio a finales de la séptima década de la vida, similar a lo descrito por otros autores ${ }^{27,28}$.

La frecuencia estimada de la etiología de los nódulos pulmonares varía sustancialmente entre los estudios, lo que refleja las diferencias entre las poblaciones de estudio y la metodología utilizada para establecer un diagnóstico. Sin embargo, aún en estudios de detección temprana en fumadores con alto riesgo de malignidad, encuentran que la gran mayoría de los nódulos identificados en la tomografía computarizada son benignos. Por ejemplo, en los estudios de Pan-Canadian Early Detection of Lung Cancer y de la British Columbia Cancer Agency, entre los 12.029 nódulos encontrados, solo 144 (1\%) eran malignos ${ }^{13}$. Teniendo en cuenta estos resultados, se podría inferir que la incidencia de nódulos malignos sea mucho menor en pacientes con riesgo medio o bajo de cáncer de pulmón.

En el presente estudio se encontró una frecuencia de nódulos malignos del 68,1\%, a pesar de la baja frecuencia de tabaquismo. Hay que tener en cuenta dos aspectos determinantes: primero, es una población de nódulos seleccionados para biopsia por considerarlos sospechosos después de ser evaluados por expertos y algunos de ellos seguidos por algún tiempo y, segundo, la mayor parte de los pacientes presentaban algún antecedente personal o familiar oncológico, lo que aumenta el riesgo de tener un nódulo maligno.

Lee y colaboradores ${ }^{27}$ muestran un predominio de aparición de nódulos pulmonares en hombres, que difiere de lo encontrado en el presente estudio, que mostró mayor frecuencia en mujeres, similar a otros estudios ${ }^{26}$.

Los márgenes espiculados y la localización de un nódulo en los lóbulos superiores se han relacionado con malignidad primaria de pulmón ${ }^{13,29}$, sin embargo, patologías inflamatorias o infecciosas también pueden verse como nódulos espiculados ${ }^{17,30,31}$. La malignidad de los nódulos espiculados incluidos en este trabajo fue del 63,5\%, pero, hay que resaltar que la característica morfológica del nódulo lobulado fue la predominante dentro del grupo de etiología maligna, con más del $84 \%$.

Además, los nódulos de tipo sólido fueron los de mayor frecuencia, en el $77 \%$ de los pacientes, siendo el patrón más frecuente, tanto en los benignos como en los adenocarcinomas. El patrón en vidrio esmerilado fue observado en adenocarcinoma in situ, mínimamente invasivo y aquellos predominantemente lepídicos. Por esto la importancia de realizar una adecuada diferenciación de los nódulos, ya que el reconocimiento de una morfología subsólida, especialmente los parcialmente sólidos, tiene un riesgo significativamente 
mayor de malignidad, al compararlos con los sólidos, sobre todo, se debe tener especial atención si los subsólidos desarrollan cambios en el tamaño, o aparece o aumenta el componente sólido. Como dato relevante llamó la atención el leve predominio de los tumores neuroendocrinos sobre los escamocelulares, probablemente porque se trató del estudio de nódulos pulmonares periféricos en su mayoría ${ }^{32}$.

En un paciente con patología maligna extratorácica conocida, la probabilidad de malignidad de un nódulo pulmonar identificado en una radiografía de tórax es de aproximadamente el $25 \%{ }^{33}$. Estos nódulos generalmente no son considerados como incidentales, ya que se encuentran en el seguimiento de otra neoplasia que potencialmente puede dar metástasis al pulmón. En nuestro estudio identificamos un predominio de nódulos pulmonares metastásicos de carcinoma gastrointestinal y mamario, que son dos de las patologías malignas que progresan muy frecuentemente con diseminación pulmonar. Clásicamente, los nódulos metastásicos se han descrito como nódulos múltiples y de bordes lisos, y en este estudio, 20 de los 25 pacientes con nódulos metastásicos (77\%), tuvieron esas características.

La causa más común de nódulos pulmonares benignos incluye infecciones granulomatosas y tumores benignos, como hamartomas, y entre las causas menos comunes están las vasculares y las lesiones inflamatorias ${ }^{28}$. En nuestro estudio fueron identificados más frecuentemente nódulos benignos de etiología inflamatoria e infecciosa, con menor proporción de tumores benignos. De igual manera, los contornos de los nódulos benignos se vieron mayormente representados por contornos lisos y espiculados, lo que concuerda con lo reportado por Vega y colaboradores, en un estudio de población chilena ${ }^{28}$.

La sensibilidad diagnóstica de la broncoscopia para obtener diagnóstico histológico en un nódulo pulmonar varía de 20 a $80 \%$, dependiendo del tamaño del nódulo, su proximidad al árbol bronquial y la prevalencia de cáncer en la población de estudio. Para nódulos que miden menos de 1,5 cm de diámetro, la sensibilidad es del $10 \%$ y para los que tienen de 2 a $3 \mathrm{~cm}$, del 40 a $60 \%{ }^{31}$. Schwarz y colaboradores lograron obtener un $41 \%$ de éxito diagnóstico mediante biopsias por broncoscopia en adultos con nódulo pulmonar solitario. En un metaanálisis de 54 estudios, analizaron el rendimiento diagnóstico de la biopsia transbronquial en 7285 nódulos, la cual fue de 70,6 \%, y este rendimiento fue superior en lesiones malignas mayores de $2 \mathrm{~cm}$, adyacentes a un bronquio ${ }^{17}$. En nuestro estudio, para la biopsia transbronquial, se obtuvo un rendimiento del $50 \%$, siendo superior en nódulos mayores de $2 \mathrm{~cm}$. No obstante, hay que tener presente que la muestra de biopsias transbronquiales es pequeña, lo que limita el análisis de los resultados. Ahora bien, en la práctica clínica consideramos que se hacen muy pocas biopsias transbronquiales, lo cual hace que la experiencia de los operadores también sea limitada, y puede impactar en los resultados.

En las biopsias percutáneas, la sensibilidad y especificidad reportada por Zhang y colaboradores $^{32}$ fueron del 95 al $100 \%$ y del 70 al $93 \%$, respectivamente. Este porcentaje de efectividad es similar al de estudios más recientes, como el reportado por Kiranantawat ${ }^{33}$, con una sensibilidad y especificidad diagnóstica para nódulos malignos de 95 y 100 \%, respectivamente. Aunque no tuvimos tan buenos resultados en nuestro estudio, sí apreciamos que al igual que las biopsias transbronquiales, el tamaño del nódulo y la experiencia del operador juegan un papel fundamental para obtener un mejor rendimiento diagnóstico.

Lo que es importante anotar es que de los 6 casos en que se realizó biopsia quirúrgica por un reporte de biopsia percutánea o transbronquial negativa para malignidad, en 3 casos (50 \%) tenían lesiones malignas y los tres restantes tumores benignos que igualmente requerían tratamiento, por eso es muy importante recalcar que una biopsia no diagnostica no puede ser malinterpretada como benigna, sino que hay que insistir en otra técnica hasta obtener un diagnóstico preciso.

La mortalidad por cáncer de pulmón podría reducirse con pruebas de detección temprana. Con el National Lung Cancer Screening Trial (NLST), se evidenció que la TC seriada redujo la mortalidad 
por cáncer de pulmón en un $20 \%$ en pacientes de alto riesgo. Los nódulos pulmonares, que fueron detectados como hallazgo incidental hasta en el $25 \%$ de los individuos en el NLST, son un ejemplo del beneficio de estos programas de tamización ${ }^{34}$. Es importante realizar en nuestro país mayores esfuerzos para lograr instaurar estos programas de tamización, que ayuden a la identificación de nódulos pulmonares que corresponden a carcinoma primario pulmonar en etapa temprana y por ende lograr reducción de la mortalidad por cáncer de pulmón. Es importante anotar que ninguno de los nódulos de este estudio fue encontrado en un programa de tamización en pacientes de alto riesgo para cáncer de pulmón, todos fueron pacientes en quienes la TC se realizó por una indicación específica, seguimiento de una neoplasia o incidentalmente en el estudio de un síntoma o de otra patología concomitante.

\section{Conclusiones}

El estudio de los nódulos pulmonares brinda información relevante que orienta sobre los diagnósticos más frecuentes en nuestra población, cuando se estudian nódulos sospechosos encontrados incidentalmente o en el seguimiento de otro tumor.

La mayoría de los nódulos en este estudio fueron de etiología maligna, diagnosticado en un 68,1 \% de las muestras analizadas histológicamente, ya que la población se limitó a nódulos considerados sospechosos y se enviaron a diagnóstico histológico, en una población que en más de un 50\% había tenido ya un tumor primario extrapulmonar.

La información obtenida, deberá servir como motivación para estudios futuros prospectivos, multicéntricos, más robustos, con una mayor cantidad de pacientes, donde se puedan analizar otras variables no incluidas en este estudio por su carácter retrospectivo, para poder conocer con mayor certeza las características de los nódulos que estamos sometiendo a diagnóstico histológico en nuestro medio. Se hace necesaria la implementación de métodos de tamización para cáncer de pulmón con estrategias que han mostrado la reducción en la mortalidad en la población susceptible.

\section{Cumplimiento de normas éticas}

Consentimiento informado. Según la resolución 8430 de 1993 se trata de una investigación sin riesgo debido a que se presentan datos retrospectivos que no modifican la conducta del paciente, por lo que no requiere diligenciamiento de consentimiento informado.

El estudio fue realizado posterior a la aprobación del protocolo por parte de la comisión de investigación y el comité de ética institucional. Se realizó en conformidad con la declaración de Helsinki de 1975, promulgada por la Asociación Médica mundial y la observancia de la Resolución Nº008430 de 1993 del Ministerio de Salud. La información estuvo bajo la custodia del investigador principal y el protocolo de investigación adherido a la ley estatutaria de habeas data.

Conflicto de interés. Los autores del estudio declararon no presentar conflictos de interés.

Financiación. El estudio fue financiado con recursos propios de los autores.

\section{Contribución de los autores}

Adquisición de datos, concepción, análisis y diseño del estudio: Stella Isabel Martínez, Alexander Fernández, Angie Carolina Riscanevo y Laura Escobar.

Análisis, revisión crítica e interpretación de datos: Stella Isabell Martínez y Alexander Fernández.

Aprobación final del manuscrito: Stella Isabel Martínez, Alexander Fernández, Angie Carolina Riscanevo y Laura Escobar.

\section{Referencias}

1. Siegel R, Naishadham D, Jemal A. Cancer statistics, 2012. CA Cancer J Clin. 2012;62:10-29. https://doi.org/10.3322/caac.20138

2. World Health Organization. International Agency for Research on Cancer. Cancer today. Fecha de consulta: 18 de febrero de 2021. Disponible en: https://gco.iarc.fr/today/home

3. Ministerio de Salud y Protección Social. Guía de práctica clínica para la detección temprana, diagnóstico, estadificación y tratamiento del cáncer de pulmón. Guía para profesionales de la salud. Bogotá D.C; 2014 p. 2014 - Guía No. 36. Fecha de consulta: 18 de Enero de 2021. Disponible en: https://www.sispro.gov.co/ observatorios/oncancer/Paginas/publicaciones-interes-piel-no-melanoma.aspx

4. Bach PB, Mirkin JN, Oliver TK, Azzoli CG, Berry DA, Brawley OW, et al. Benefits and harms of CT screening for lung cancer. JAMA. 2012;307:2418-29. https://doi.org/10.1001/jama.2012.5521 
5. Sihoe ADL, Cardillo G. Solitary pulmonary ground-glass opacity: is it time for new surgical guidelines? European Journal of Cardio-Thoracic Surgery. 2017;52:848-51. https://doi.org/10.1093/ejcts/ezx211

6. The National Lung Screening Trial Research Team. Reduced lung-cancer mortality with low-dose computed tomographic screening. N Engl J Med. 2011;365:395409. https://doi.org/10.1056/NEJMoa1102873

7. Bray F, Ferlay J, Soerjomataram I, Siegel RL, Torre LA, Jemal A. Global cancer statistics 2018: GLOBOCAN estimates of incidence and mortality worldwide for 36 cancers in 185 countries. CA Cancer J Clin. 2018;68:394424. https://doi.org/10.3322/caac.21492

8. Houghton AM. Mechanistic links between COPD and lung cancer. Nat Rev Cancer. 2013;13:233-45. https://doi.org/10.1038/nrc3477

9. Hammer MM, Hatabu H. Subsolid pulmonary nodules: Controversy and perspective. Eur J Radiol Open. 2020;7:100267. https://doi.org/10.1016/j.ejro.2020.100267

10. Wang W, Xie M, Dou S, Cui L, Zheng CY, Xiao W. The link between chronic obstructive pulmonary disease phenotypes and histological subtypes of lung cancer: a case - control study. International Journal of COPD. 2018;13:1167-75.

https://doi.org/10.2147/COPD.S158818

11. MacMahon H, Naidich DP, Goo JM, Lee KS, Leung ANC, Mayo JR, et al. Guidelines for management of incidental pulmonary nodules detected on CT images: From the Fleischner Society 2017. Radiology. 2017;284:228-43. https://doi.org/10.1148/radiol.2017161659

12. Chen D, Dai C, Kadeer X, Mao R, Chen Y, Chen C. New horizons in surgical treatment of ground-glass nodules of the lung: experience and controversies. Ther Clin Risk Manag. 2018;14:203-11. https://doi.org/10.2147/TCRM.S152127

13. Takasugi J, Godwin J. The Solitary Pulmonary Nodule: Radiologic Assessment. In: Sperber M (eds). Radiologic Diagnosis of Chest Disease. Springer, London. https://doi.org/10.1007/978-1-4471-0693-7_32

14. McWilliams A, Tammemagi M, Mayo J, Roberts H, Liu G, Soghrati K et al. Probability of Cancer in Pulmonary Nodules Detected on First Screening CT. N Engl J Med. 2013;369:910-9.

https://doi.org/10.1056/NEJMoa1214726

15. Gould MK, Donington J, Lynch WR, Mazzone PJ, Midthun DE, Naidich DP, Wiener RS. Evaluation of individuals with pulmonary nodules: when is it lung cancer? Diagnosis and management of lung cancer, 3rd ed: American College of Chest Physicians evidence-based clinical practice guidelines. Chest. 2013;143:e93S-e120S. https://doi.org/10.1378/chest.12-2351

16. Seo JB, Im JG, Goo JM, Chung MJ, Kim MY. Atypical pulmonary metastases: Spectrum of radiologic findings. Radiographics. 2001;21:403-17.

https://doi.org/10.1148/radiographics.21.2.g01mr17403
17. Ost D, Fein AM, Feinsilver SH. The Solitary Pulmonary Nodule. N Engl J Med. 2003;348:2535-42. https://doi.org/10.1056/NEJMcp012290

18. Barrio JL, Suarez M, Rodriguez JL, Saldana MJ, Pitchenik AE. Pneumocystis carinii pneumonia presenting as cavitating and noncavitating solitary pulmonary nodules in patients with the acquired immunodeficiency syndrome. Am Rev Respir Dis. 1986;134:1094-6. https://doi.org/10.1164/arrd.1986.134.5.1094

19. Echeverri A, Long RF, Check W, Burnett CM. Pulmonary dirofilariasis. The Ann Thorac Surg. 1999;67:201-2. https://doi.org/10.1016/s0003-4975(98)01060-1

20. Guo W, Zhao YP, Jiang YG, Wang RW, Ma Z. Surgical treatment and outcome of pulmonary hamartoma: a retrospective study of 20-year experience. Journal of Experimental \& Clinical Cancer Research. 2008;27:8. https://doi.org/10.1186/1756-9966-27-8

21. Dai J, Yu G, Yu J. Can CT imaging features of groundglass opacity predict invasiveness? A meta-analysis. Thoracic Cancer. 2018;9:452-8. https://doi.org/10.1111/1759-7714.12604

22. Maj GT, Gracey DR, Byrd RB. The management and evaluation of the solitary pulmonary nodule. Chest. 1974;66:236-9. https://doi.org/10.1378/chest.66.3.236

23. Gruden JF, Ouanounou S, Tigges S, Norris SD, Klausner TS. Incremental benefit of maximum-intensity-projection images on observer detection of small pulmonary nodules revealed by multidetector CT. Am J Roentgenol. 2002;179:149-57. https://doi.org/10.2214/ajr.179.1.1790149

24. Gould MK, Ananth L, Barnett PG. A clinical model to estimate the pretest probability of lung cancer in patients with solitary pulmonary nodules. Chest. 2007;131:383-8. https://doi.org/10.1378/chest.06-1261

25. Gohagan J, Marcus P, Fagerstrom R, Pinsky P, Kramer B, Prorok P, for The Lung Screening Study Research Group. Baseline findings of a randomized feasibility trial of lung cancer screening with spiral CT scan vs chest radiograph. The Lung Screening Study of the National Cancer Institute. Chest. 2004;126:114-21. https://doi.org/10.1378/chest.126.1.114

26. Gurney JW. Determining the likelihood of malignancy in solitary pulmonary nodules with Bayesian analysis. Part I. Theory. Radiology. 1993;186:405-13. https://doi.org/10.1148/radiology.186.2.8421743

27. Lee JE, Jeong WG, Kim YH. Differentiation of primary lung cancer from solitary lung metastasis in patients with colorectal cancer: a retrospective cohort study. World Journal of Surgical Oncology. 2021;19:28. https://doi.org/10.1186/s12957-021-02131-7

28. Vega J, Lazo D, Undurraga F, Clavero JM, Rodríguez P. Caracterización de nódulos pulmonares resecados. Experiencia de manejo por un programa multidisciplinario. Rev méd Chile. 2018;146:1261-8. http://dx.doi.org/10.4067/S0034-98872018001101261 
29. Snoeckx A, Reyntiens P, Desbuquoit D, Spinhoven MJ, Van Schil PE, van Meerbeeck JP, Parizel PM. Evaluation of the solitary pulmonary nodule: size matters, but do not ignore the power of morphology. Insights into Imaging. 2018;9:73-86.

http://doi.org/10.1007/s13244-017-0581-2

30. Ost D, Fein A. Management strategies for the solitary pulmonary nodule. Curr Opin Pulm Med. 2004;10:2728. http://doi.org/10.1097/01.mcp.0000130322.11513.c8

31. Oke JL, Pickup LC, Declerck J, Callister ME, Baldwin D, Gustafson J, et al. Development and validation of clinical prediction models to risk stratify patients presenting with small pulmonary nodules: a research protocol. Diagn Progn Res. 2018;2:22.

https://doi.org/10.1186/s41512-018-0044-3
32. Zhang M, Kono M. Solitary pulmonary nodules: evaluation of blood flow patterns with dynamic CT. Radiology. 1997;205:471-8.

https://doi.org/10.1148/radiology.205.2.9356631

33. Kiranantawat N, McDermott S, Fintelmann FJ, Montesi SB, Price MC, Digumarthy SR, Sharma A. Clinical role, safety and diagnostic accuracy of percutaneous transthoracic needle biopsy in the evaluation of pulmonary consolidation. Respir Res. 2019;20:23. https://doi.org/10.1186/s12931-019-0982-5

34. Clark ME, Young B, Bedford LE, das Nair R, Robertson JFR, Vedhara $\mathrm{K}$, et al. Lung cancer screening: does pulmonary nodule detection affect a range of smoking behaviours? J Public Health (Oxf). 2018;41:600-8. https://doi.org/10.1093/pubmed/fdy158 